\title{
Better medicines for neonates: Improving medicine development, testing, and prescribing
}

\author{
Karel Allegaert* \\ Intensive Care and Department of Pediatric Surgery, Erasmus MC-Sophia Children's Hospital, Rotterdam, The Netherlands \\ Neonatology, Erasmus MC-Sophia Children's Hospital, Rotterdam, The Netherlands \\ Department of Development and Regeneration, KU Leuven, Leuven, Belgium.
}

\section{A R T I C L E I N F O}

\section{Keywords:}

Newborn

Clinical pharmacology

Pharmacodynamics

Pharmacokinetics

Safety

Medicine development

\begin{abstract}
A B S T R A C T
Pharmacotherapy is a powerful tool to improve the outcome of neonates. Unfortunately, the potential health impact of pharmacotherapy in neonates remains underexplored. This necessitates a structured approach to go beyond the current practice of trial and error, reflected in off-label prescription. The existing regulatory framework hereby provides a structure to reflect about aspects like pharmacokinetic models for dose selection and outcome assessment, including long-term safety. Future medicine development should also be driven by neonatal needs, diseases and pathophysiology, since surfactant is the latest product developed for preterm neonates. The potential impact is illustrated by ongoing repurposing (propranolol, allopurinol, erythropoietin, Insulin-like Growth Factor-1) projects.

Clinical researchers will be crucial to close the knowledge gap by developing dose selection tools and outcome assessment tools and by exploring pathophysiological mechanisms. The final step of such a structured approach cycle is the subsequent translation of accumulated knowledge into improved prescribing.
\end{abstract}

1. Pharmacotherapy in the newborn: how to get beyond trial and error?

When health care professionals administer a medicine to a newborn, it is with the intention to provide effective relief for a given indication (e.g. infection, retinopathy of prematurity, pain), while still avoiding disproportional side-effects. Clinical pharmacology aims to predict the effects of such interventions, applying pharmacokinetics (PK) and pharmacodynamics (PD) as mathematical concepts to generate predictions, including confidence intervals. PK (ADME, absorption, distribution and elimination, through either metabolism or renal elimination) estimates the relationship between a concentration at a specific site (e.g. cerebrospinal fluid, blood compartment) with time ('what the body does to the medicine'). PD aims to estimate both the effects and sideeffects of a given medicine in relation to a given concentration ('what the medicine does to the body') [1,2]. Because of the fast maturational changes in neonatal life with age (postnatal, postmenstrual) and weight (birth weight, current weight) as main drivers (covariates) of this maturation, PK and subsequent PD display extensive between and withinindividual variability $[1,2]$.

The physiology-related maturation in ADME processes is reflected in changes in body composition, protein binding and subsequent compartment size changes. All phase I (e.g. cytochromes) and phase II (e.g. glucuronidation) metabolic processes of medicines mature in an enzyme specific pattern, while renal function [glomerular filtration rate (GFR), tubular absorption/excretion] also display age-dependent clearance [3]. Age-dependent PD differences are much less explored, but also relate to age and population-specific effects (e.g. caffeine to treat neonatal apnoea, oxygen and retinopathy of prematurity, cerebral palsy related to postnatal steroids) [3]. Consequently, dosing of medicines in young infants should be based on integrated knowledge concerning the specific diseases to be treated, the physiological characteristics of the newborn receiving the medicine, and the PK-PD parameters of the medicine. This makes clinical research on pharmacotherapy in neonates relevant, but also more difficult to perform $[1,2,3]$.

Unfortunately, the potential health impact of neonatal pharmacotherapy remains underexplored. It is still very common practice to administer medicines outside their market authorization (indication in this population, off-label). Unlicensed prescription refers to the use of an approved medicine in an unapproved formulation. The most recent (2015) meta-analysis on unlicensed and off-label medicine prescription practices reconfirmed that this practice is still widespread in pediatrics, and that the youngest age category, i.e. (pre)term neonates are exposed

\footnotetext{
* Corresponding author at: Department of Development and Regeneration, KU Leuven, Herestraat 49, 3000 Leuven, Belgium.

E-mail address: karel.allegaert@uzleuven.be.
} 
Table 1

Number of studies and proportion of clinical studies as retrieved on www.clinicaltrials. gov (30 July 2017), using either no specific search criteria (all studies) or retrieved when 'child', 'infant' or 'newborn' were entered.

\begin{tabular}{lllll}
\hline & All studies & 'child' & 'infant' & 'newborn' \\
\hline Worldwide & 250,710 & $55,942(23 \%)$ & $8603(3 \%)$ & $5451(2 \%)$ \\
United States & $103,757(41 \%)$ & $23,664(42 \%)$ & $3592(42 \%)$ & $2038(38 \%)$ \\
Europe & $70,579(28 \%)$ & $12,145(22 \%)$ & $2026(25 \%)$ & $1541(28 \%)$ \\
Canada & $17,142(8 \%)$ & $4180(7 \%)$ & $784(9 \%)$ & $409(8 \%)$ \\
Pacific & $6216(2.5 \%)$ & $1236(2 \%)$ & $291(3 \%)$ & $102(2 \%)$ \\
South America & $8314(3 \%)$ & $1884(3.5 \%)$ & $263(3 \%)$ & $167(3 \%)$ \\
\hline
\end{tabular}

most commonly (100\%) to unlicensed or off-label medicines [4]. Although off-label is not always equal to off-knowledge, this practice does result in the fact that health care professionals commonly lack the availability or access to crucial information to make the best possible, informed decision and to discuss options with parents: do we accept to continue to use this trial and error approach? It is not because we have been using a medicine for even decades that we know enough about the medicine and how to use a given medicine effectively and safe. Oxygen or postnatal steroids may hereby serve as relevant illustrations to neonatologists. Finally, off-label practices are only one side of the coin, as this also reflects the fact that the potential health impact of neonatal pharmacotherapy remains underexplored [5].

There is a legal framework and ongoing initiatives to generate knowledge on neonatal pharmacotherapy to improve this setting. To quantify and put these activities into perspective, studies in infants and in newborns cover 3 and $2 \%$ of all registered studies respectively, with a similar spread throughout different regions (Table 1). Unfortunately, $42 \%$ of pediatric studies ( $n=44,2007-2014$, submitted to the Food and Drug Administration (FDA) failed to document efficacy ( $n=39$, $86 \%)$ or safety $(n=7,16 \%)$ due to inaccurate dosing $(n=10)$ or failure to sufficiently consider the differences in the pediatric vs adult disease $(n=8)$ [6]. Neonatal pharmacotherapy is lagging even further behind when compared to other pediatric populations [7]. Stiers and Ward recently reported that only a limited number of label changes (24/406, 6\%) included labelling changes for neonates (1997-2010, FDA), claiming that newborns were one of the last therapeutic orphans to be adopted. This seems to relate to inaccurate dose selection and insufficient assessment of neonatal pathophysiology. As additional weaknesses, the majority of studies were single center studies (58\%), and industry was sponsor in a limited number $(23 \%)$ of the registered trials [8]. Additionally, the traditional control trial design, especially for the extremely preterm neonates is often perceived not to be feasible [9]. However, in a recent analysis on studies in neonates registered within the clinicaltrials.gov application, Desselas et al. concluded that placebo versus drug randomized controlled trials (RCT) represent 34 $(146 / 423) \%$ of the registered neonatal trials with steroids, erythropoietin and nitric oxide as the most commonly evaluated medicines [10].

The existing regulatory framework hereby provides a structure to reflect about aspects like PK models for dose selection and outcome assessment, including long-term safety. Future medicine development should also be driven by neonatal needs, diseases and pathophysiology, since surfactant is the latest product developed for preterm neonates. The potential impact is illustrated by ongoing repurposing (propranolol, Insulin-like Growth Factor-1, allopurinol) projects $[1,12,13]$. Contributions of health care professionals active in neonatal care will be crucial to enable the best use of the regulatory framework, to generate the knowledge needed to develop dose selection tools and outcome assessment tools and to explore pathophysiological mechanisms. The same health care professionals will also be crucial to enable the final step of such a structured approach cycle: the subsequent translation of the accumulated knowledge into improved prescribing.

\section{Current regulatory framework for medicine development program applied to neonates}

Both the FDA and European Medicines Agency (EMA) have converted pediatric legislation to initiatives to optimize medicine evaluation in pediatric populations with the intention to result in label changes, including in neonates as recently discussed in this journal $[5,14]$. Such efforts should be based on the neonatal study decision tree [Fig. 1] as applied by these authorities to assess neonatal medicine development plans [15]. Such a medicine development plan can be defined as the aggregate of individual studies conducted in the course of the product development cycle, and can include studies on efficacy, safety, PK or PD, and tolerability [6].

As mentioned earlier, diseases may be specific to neonates, the impact of immaturity and rapid developmental changes in early life is important, and medicines may have short and long-term effects including developmental toxicity. Consequently, a neonatal study decision tree is useful to reflect about potential scenarios. Scenario 1 is appropriate when extrapolation of the exposure-response is possible and the dose-exposure (PK) is to be documented (e.g. antibiotics for sepsis, antifungals), including safety. Scenario 2 or 3 are appropriate

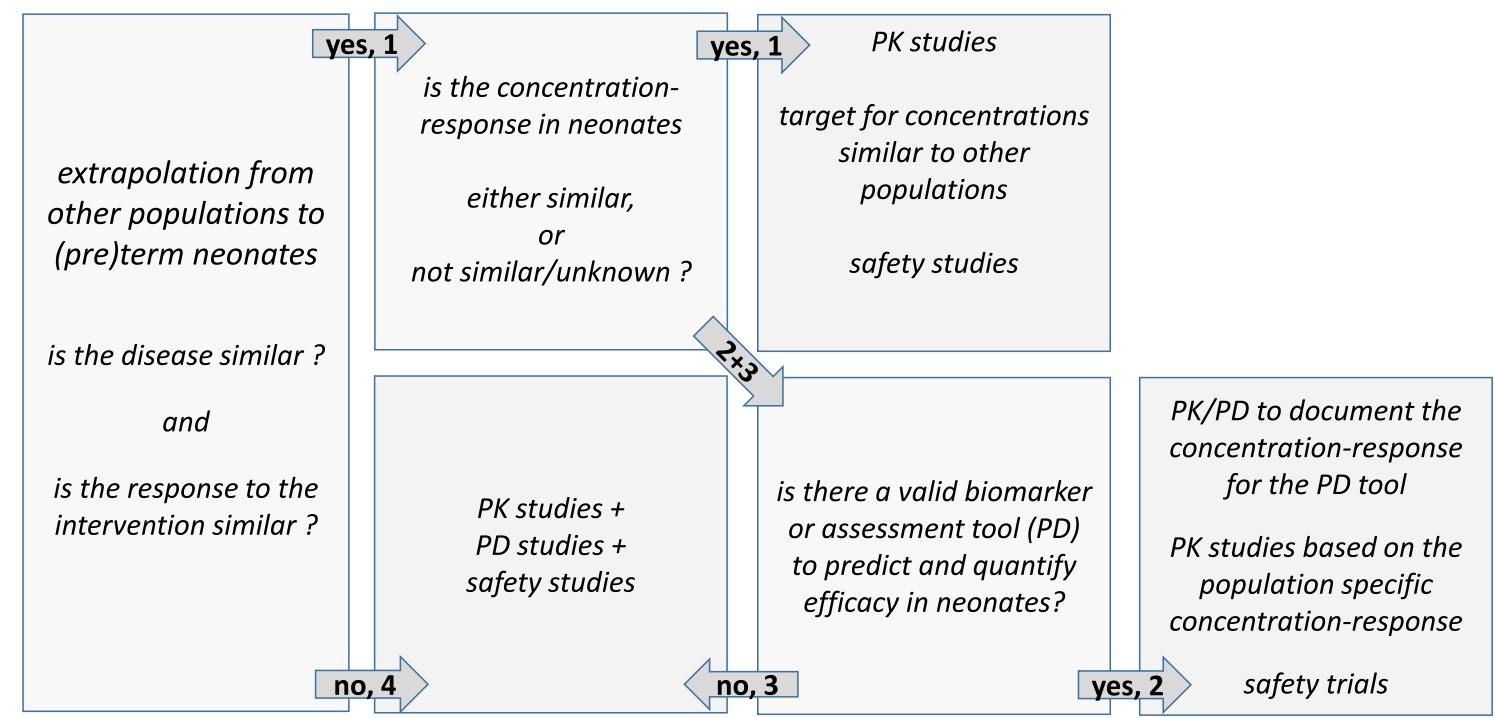

Fig. 1. Pediatric medicine study decision tree as applied by the authorities to assess neonatal medicine development plans, adapted from Pons and Manolis [15]. 
when the exposure-response is known, but extrapolation is uncertain (e.g. necrotizing enterocolitis, meropenem studies). Additional classification (scenario 2 vs 3 ) depends on the availability of a biomarker or assessment tool (e.g. Electro-encephalography for seizures, cardiac ultrasound for patent ductus arteriosus, hypotension). In the setting of a disease or disease progression that is specific to neonates, a full program (scenario 4) should be developed (e.g. hyaline membrane disease, bronchopulmonary dysplasia, retinopathy of prematurity) $[3,15]$.

As mentioned earlier, Stiers and Ward documented that the majority of the studies failed to establish efficacy in neonates [7]. It is uncertain whether the lack of efficacy is accurate, or reflects study design issues including disease characteristics in the neonate, medicine properties (dose, formulation), or the inability of endpoints to reflect clinical meaningful outcomes. To raise the likelihood that such a medicine development cycle result in labelling for a given indication in neonates, it is crucial that stakeholders (clinical researchers, industry, parent advocacy groups, agencies) collaborate to provide the pieces of the puzzle to ultimately provide safe and effective medicines for neonates.

These pieces include - but are not limited to - study design related issues: the extent and type of outcome measures, the use of phase 2 before phase 3 studies as dose seeking, the use of master protocols, or safety assessment). Using a systematic review on 119 published papers on neonatal RCT, Zhang et al. documented that the postulated median relative risk reduction in these studies was $40-50 \%$. Consequently, modest but still clinical relevant treatment effects can been missed [16]. Poor outcome selection remains an important cause of study failure. Standardization of core outcomes sets and standardize reporting of these sets supports study design, interpretation and comparison between different studies in cross-study evaluations and meta-analysis [17]. Similar, standardization of data items collected in neonatal database will facilitate linkage of datasets and cross talk [18]. At least as relevant of standardization, is the availability of robust case definitions like for bronchopulmonary dysplasia or necrotizing enterocolitis $[19,20]$. Phase 2 studies or therapeutic exploratory studies may be very useful to raise the likelihood of a valid study design - including dose seeking - before conducting the pivotal phase 3 efficacy trials and are common practice in 'adult' product development [5]. To assess safety or tolerance, the earlier mentioned standardization of core outcome sets and data sets can be used, further extended with e.g. age-adapted references for laboratory values, growth patterns or neurodevelopment to differentiate safety issues from confounding disease processes and concomitant medication exposure.

\section{Towards a well educated guess to improve dose selection in neonates}

Medicine development in neonates remains challenging, but there is growing knowledge on modeling and simulation to support health care professionals in decisions on dose selection. Medicine modeling efforts are effective when these efforts support any decision on individualized dosing in the clinical setting as well as on study conduct. Agencies also strongly support the use of modeling and simulation methods for any pediatric medicine development program [21]. The major advantage is that a study hereby becomes confirmatory instead of exploratory and makes a robust conclusion more likely by using validating predictions approaches from carefully constructed predictive models (well educated guess instead of trial and error). These approaches also further reduce the burden for individual patients, especially when combined with sparse sampling techniques. The relevance is reflected in the earlier mentioned high incidence of study failure due to inaccurate dose selection [7]. There are in essence two different approaches, either empiric, exploratory semi-physiological model building or physiologically based-pharmacokinetics (PB-PK) [1,3].

Clinicians can contribute to both modeling processes by data sharing, including collection of the relevant covariates, or by generating data on pathophysiology, or by contributing to prospective validation efforts. Hundreds of compound specific clinical pharmacology studies have been conducted in pediatric populations, including neonates. These compound specific observations can be used to develop semiphysiological models, as illustrated by the use of a neonatal amikacin covariate model to predict ontogeny of other medicines eliminated by glomerular filtration in neonates [22]. Similar, an age-staggered approach is commonly used with the intention to adapt prior data from older pediatric patients and adults to inform the dose in younger patients. Wang et al. hereby illustrated that a model with data from infants ( $<2$ years) was more accurate (lower bias) to predict neonatal clearance and dosing [23]. In the absence of such data, models tended to overpredict neonatal clearance. PB-PK models aim to translate physical, physiological, anatomic and/or chemical descriptions of processes involved in medicine disposition to predict concentration/time or concentration/effect patterns. PB-PK modeling provides an approach to incorporate different types of information (physicochemical properties, preclinical data, clinical data) to estimate age-specific PK and to turn data into knowledge [2].

The concepts behind these model building processes also matter to clinical researchers. Population PK approaches generate estimations of mean and variances through merging observations. Consequently, it is advisable to include data on covariates like age (gestational, postnatal, postmenstrual), weight (birth, current), renal function, albumin, concomitant medication, or co-morbidity in PK/PD studies: good data have their relevance beyond compound specific estimates [2]. Similar, the available knowledge on developmental physiology is still too limited, particularly in preterm neonates and such data can be used to support PB-PK model development. It is up to clinical researchers to generate these data. Finally, prospective validation is needed. As illustrated by Wilbaux et al., only $20 \%$ of the models on primarily renally eliminated antibiotics in neonates were validated based on an external dataset [24]. Cross-validation of published vancomycin PK models in neonates from different units failed and this failure could be explained by different analytical methods used in the initial model to measure serum creatinine and vancomycin [25].

\section{Medicine development tailored to or driven by neonatal needs}

Future medicine development should also be driven by neonatal needs, diseases and pathophysiology. To the very best of our knowledge, surfactant is the latest product specifically developed for preterm neonates, hopefully not the last. This is important to understand the pathophysiology of neonatal disease processes not just at time of birth but with respect to postnatal adaptive responses. This is especially true with respect to safety and understanding potential off-target effects of medicines in the developing neonate [5]. Common illustrations are e.g. glomerulogenesis during fetal or postnatal age in extreme preterm neonates, fetal to postnatal growth and body composition or persistent pulmonary hypertension of the newborn.

An emerging concept to facilitate medicine development is repurposing, i.e. the use of 'old' medicines for new indications, avoiding the need for time- and resource-intensive toxicity studies [26]. The potential relevance to neonates can be illustrated by repurposing projects related to propranolol (hemangioma, retinopathy of prematurity), allopurinol (asphyxia) or Insulin-like Growth Factor-1 (retinopathy of prematurity) $[11,12,13]$. Insulin-like Growth Factor 1 has multisystem effects on fetal and preterm infant development, reflecting the relevance to understand potential off-target positive or negative effects and the relevance of safety assessment [13].

\section{Prescribing: do we practice what we preach?}

The next step of the cycle is the subsequent translation of knowledge into improved prescribing. This includes the shift from efficacy (effects under well controlled trial conditions) to effectiveness (effects of medicines under routine clinical care). Besides labelling, inappropriate 
formulations, (poly)pharmacy, immature organ function or co-morbidity further raise the risk for adverse drug reactions in neonates [27]. In the medicine product cycle as currently used, post marketing studies became even more relevant since safety issues may only arise during routine clinical care $[3,27]$. The earlier mentioned standardization of core outcome sets, standardization of data items and the use of relevant case definitions may also be very supportive to generate robust safety information. Access to dosing regimens is another issue, and can be secured through a pediatric medicine formulary. Such an approach has recently provided dosing guidelines based on best available evidence from registration data, investigator-initiated research, professional guidelines, clinical experience and consensus, with 'maintenance' activities through pre-planned re-assessment of the available information [28]. We should be aware that labelling provide us with the access to crucial information to make the best possible, informed decision and to discuss options with parents.

\section{Conclusions}

Clinical researchers remain crucial as contributors to improve neonatal pharmacotherapy. This relates to generate the knowledge needed to develop dose selection tools and outcome assessment tools, to explore pathophysiological mechanisms and to improve our prescribing practices.

Key guidelines

- The potential health impact of pharmacotherapy in neonates remains underexplored.

- Labelling provide us with the access to crucial information to make the best possible, informed decision and to discuss options with parents.

- The existing regulatory framework provides a structure to reflect about neonatal medicine development plans and how clinical researchers can contribute to improved pharmacotherapy and labelling.

\section{Research directions}

- Generate the knowledge needed to develop dose selection tools, outcome assessment tools and to explore pathophysiological mechanisms in search of targets.

- Contribute to standardization of core outcome sets, standardization of data items and the use of relevant case definitions to improve study conduct and interpretation.

- Contribute to more accurate prescription of medicines by contributing to safety data (using the same standardization and case definitions) and through contribution to the development and maintenance of drug formularies.

\section{Acknowledgements}

Karel Allegaert has been supported by the Fund for Scientific Research, Flanders (fundamental clinical investigatorship 1800214N), and his research activities are further facilitated by the agency for innovation by Science and Technology in Flanders (IWT) through the SAFEPEDRUG project (IWT/SBO 130033).

\section{Conflicts of interest}

The author reports no conflict of interest.

\section{References}

[1] K. Allegaert, J. van den Anker, Neonatal drug therapy: the first frontier of therapeutics for children, Clin. Pharmacol. Ther. 98 (2015) 288-297.

[2] A. Smits, A. Kulo, J.N. de Hoon, K. Allegaert, Pharmacokinetics of drugs in neonates: pattern recognition beyond compound specific observations, Curr. Pharm. Des. 18 (2012) 3119-3146.

[3] R.M. Ward, D. Benjamin, J.S. Barrett, et al., Safety, dosing, and pharmaceutical quality for studies that evaluate medicinal products (including biological products) in neonates, Pediatr. Res. 81 (2017) 692-711.

[4] J. Magalhaes, A.T. Rodrigues, F. Roque, A. Figueiras, A. Falcao, M.T. Herdeiro, Use of off-label and unlincenced drugs in hospitalised paediatric patients: a systematic review, Eur. J. Clin. Pharmacol. 71 (2015) 1-13.

[5] M.A. Turner, Neonatal drug development, Early Hum. Dev. 87 (2011) 763-768.

[6] J.D. Momper, Y. Mulugeta, G.J. Burckart, Failed pediatric drug development trials, Clin. Pharmacol. Ther. 98 (2015) 245-251.

[7] J.L. Stiers, R.M. Ward, Newborns, one of the last therapeutic orphans to be adopted, JAMA Pediatr. 168 (2014) 106-108.

[8] C. Pansieri, M. Bonati, I. Choonara, E. Jacqz-Aigrain, Neonatal drug trials: impact of EU and US paediatric regulations, Arch. Dis. Child. Fetal Neonatal Ed. 99 (2014) F438.

[9] J.R. Wiles, A.A. Vinks, H. Akinbi, Federal legislation and the advancement of neonatal drug studies, J. Pediatr. 162 (2013) 12-15.

[10] E. Desselas, C. Pansieri, S. Leroux, M. Bonati, E. Jacqz-Aigrain, Drug versus placebo randomized controlled trials in neonates: a review of clinicaltrials.gov registry, PLoS One 12 (2017) e0171760.

[11] C. Léauté-Labrèze, J.I. Harper, P.H. Hoeger, Infantile haemangioma, Lancet 390 (2017) 85-94.

[12] T. Chaudhari, W. McGuire, Allopurinol for preventing mortality and morbidity in newborn infants with hypoxic-ischaemic encephalopathy, Cochrane Database Syst. Rev. 7 (2012) CD006817.

[13] A. Hellstrom, D. Ley, I. Hansen-Pupp, et al., Insulin-like growth factor 1 has multisystem effects on foetal and preterm infant development, Acta Paediatr. 105 (2016) 576-586.

[14] E. Jacqz-Aigrain, Drug policy in Europe research and funding in neonates: current challenges, future perspectives, new opportunities, Early Hum. Dev. 87 (2011) S27-S30.

[15] E. Manolis, G. Pons, Proposals for model-based paediatric medicinal development within the current European Union regulatory framework, Br. J. Clin. Pharmacol. 68 (2009) 493-501.

[16] B. Zhang, B. Schmidt, Do we measure the right end points? A systematic review of primary outcomes in recent neonatal randomized clinical trials, J. Pediatr. 138 (2001) 76-80.

[17] J. Webbe, G. Brunton, S. Ali, et al., Developing, implementing and disseminating a core outcome set for neonatal medicine, BMJ Paediatr. Open 1 (2017) e000048.

[18] Y. Statnikov, B. Ibrahim, N. Modi, A systematic review of administrative and clinical databases of infants admitted to neonatal units, Arch. Dis. Child. Fetal Neonatal Ed. 102 (2017) F270-F276.

[19] T. Isayama, S.K. Lee, J. Yang, et al., Revisiting the definition of bronchopulmonary dysplasia: effect of changing panoply of respiratory support for preterm neonates, JAMA Pediatr. 171 (2017) 271-279.

[20] C. Battersby, N. Longford, K. Costeloe, N. ModiUK Neonatal Collaborative Necrotising Enterocolitis Study Group, Development of a gestational age-specific case definition for neonatal necrotizing enterocolitis, JAMA Pediatr. 171 (2017) 256-263.

[21] J. Zisowsky, A. Krause, J. Dingemanse, Drug development for pediatric populations: regulatory aspects, Pharmaceutics 2 (2010) 364-388.

[22] R.F. De Cock, K. Allegaert, C.M. Sherwin, et al., A neonatal amikacin covariate model can be used to predict ontogeny of other drugs eliminated through glomerular filtration in neonates, Pharm. Res. 31 (2014) 754-767.

[23] J. Wang, A.N. Edginton, D. Avant, G.J. Burckart, Predicting neonatal pharmacokinetics from prior data using population pharmacokinetic modeling, J. Clin. Pharmacol. 55 (2015) 1175-1183.

[24] M. Wilbaux, A. Fuchs, J. Samardzic, et al., Pharmacometric approaches to personalize use of primarily renally eliminated antibiotics in preterm and term neonates, J. Clin. Pharmacol. 56 (2016) 909-935.

[25] W. Zhao, F. Kaguelidou, V. Biran, et al., External evaluation of population pharmacokinetic models of vancomycin in neonates: the transferability of published models to different clinical settings, Br. J. Clin. Pharmacol. 75 (2013) 1068-1080.

[26] J. Blatt, S. Farag, S.J. Corey, et al., Expanding the scope of drug repurposing in pediatrics: the children's pharmacy collaborative, Drug Discov. Today 19 (2014) 1696-1698.

[27] K. Allegaert, J.N. van den Anker, Adverse drug reactions in neonates and infants: a population-tailored approach is needed, Br. J. Clin. Pharmacol. 80 (2015) 788-795.

[28] T.M. Van der Zanden, S.N. de Wildt, Y. Liem, et al., Developing a paediatric drug formulary for The Netherlands, Arch. Dis. Child. 102 (2017) 357-361. 